\title{
VALIDACIÓN DE SEÑALES VIBRO-ACÚSTICAS PARA EL DIAGNÓSTICO DE FALLAS EN RODAMIENTOS EN UN GENERADOR SÍNCRONO
}

\section{VALIDATION OF ACOUSTIC VIBES SIGNALS TO DIAGNOSIS FAILURE ON SYNCHRONOUS GENERATOR BEARINGS}

Zulma Yadira Medrano Hurtado*

Carlos Pérez Tello*

Fecha de recepción: 26 de julio de 2016

Fecha de revisión: 9 de febrero de 2017

Fecha de aprobación: 24 de febrero de 2017

Cómo citar: Z. Y. Medrano Hurtado y C. Pérez Tello, "Validación de señales vibro-acústicas para el diagnóstico de fallas en rodamientos en un generador sincrónico," Ciencia e Ingeniería Neogranadina, vol. 27, no. 2, pp. 51-81. DOI: http://dx.doi.org/10,18359/rcin.2180

\section{RESUMEN}

En este trabajo se describe el procedimiento y las herramientas utilizados en la medición y diagnóstico de señales de vibración capturadas a través de transductores de aceleración (acelerómetros piezoeléctricos) y acústicos (micrófonos omnidireccionales). Además, se desarrolló un arreglo experimental empleando la metodología Taguchi para validar la información registrada de las señales de vibración para rodamientos sin falla y con falla artificial, respectivamente. La falla artificial consistió en una grieta producida en la jaula de un rodamiento SKF-6303-2RSH. Este método es no invasivo, ya que utiliza micrófonos para analizar la vibración, lo que representa no tener que montar ningún tipo de transductor en la máquina, además de ser sensible a fallas en la jaula.

\footnotetext{
* Ingeniera electricista; doctora en Ingeniería. Profesora del Instituto Tecnológico de Mexicali. Mexicali, Baja California, México. Correo electrónico: zulmamh@itmexicali.edu.mx, zulmamh@yahoo.com.mx. ORCID: http://orcid.org/0000-0003-0731-6529

** Ingeniero químico; doctor en Ciencias. Investigador, de la Universidad Autónoma de Baja California, MexicaIi, Baja California, México. Correo electrónico: carlosperez@uabc.edu.mx. ORCID: http://orcid.org/0000-00020683-3025
} 
Palabras clave: Metodología Taguchi, acelerómetros piezoeléctricos, micrófonos omnidireccionales, rodamiento.

\section{ABSTRACT}

This paper describes the procedure and tools used in the measurement and diagnosis of vibration signals captured by acceleration transducers (piezoelectric accelerometers) and acoustic (omnidirectional microphones). Also, An experimental arrangement was developed using the Taguchi methodology to validate the recorded information of the vibration signals for bearings without failure and with an artificial failure, respectively. The artificial fault consisted of a crack produced in the cage of a bearing SKF-6303-2RSH. This method is non-invasive since it uses microphones to analyze the vibration, which means not having to mount any transducer at the machine besides being sensitive to failures in the cage.

Keywords: Taguchi methodology, piezoelectric accelerometers, omnidirectional microphones, bearing.

\section{INTRODUCCIÓN}

Este trabajo explica estadísticamente el cambio de los transductores convencionales que se utilizan para medir la vibración que se basa en el empleo de registros a través de acelerómetros (acelerómetros piezoeléctricos), hacia un tipo de transductores distinto, pero igualmente confiable, que permite la utilización de transductores que se basan en la emisión acústica de las máquinas (micrófonos omnidireccionales), empleando la metodología Taguchi.

Los generadores síncronos producen alrededor del $99 \%$ del consumo de electricidad mundial [1], y por su importancia en la generación eléctrica global es necesaria la anticipación a la ocurrencia de fallas que puedan llegar a producir problemas indeseados. Este tipo de máquinas se hallan expuestas a un gran número de fallas y cons- tituyen el equipo más costoso del sistema eléctrico de potencia [2]-[4], lo que las sitúa en una posición determinada de carácter crítico. Las fallas en rodamientos son uno de los problemas más comunes en los generadores síncronos; aproximadamente el $40 \%$ [5]-[7] de las fallas en las máquinas eléctricas rotativas.

La forma convencional de análisis y diagnóstico de falla en los rodamientos se ha basado en el empleo de registros de señales de vibración, que juegan en la actualidad un papel importante en el mantenimiento predictivo para analizar su comportamiento, pero que presentan la desventaja de tener que montar el transductor en la máquina. Esto significa la necesidad y oportunidad de contar con una metodología distinta, pero igualmente confiable, que permita la utilización de otro tipo de señal. Lo anterior propo- 
ne un sistema de diagnóstico basado en la emisión acústica de las máquinas, utilizando micrófonos como transductores.

Como se conoce, cualquiera que sea la causa de la vibración en un generador síncrono, esta resulta en una emisión acústica. El presente trabajo propone un método de diagnóstico basado en la emisión acústica de un generador síncrono. Dicha técnica detecta cambios acústicos internos provocados por fenómenos como el surgimiento y crecimiento de grietas, el desprendimiento de pequeños fragmentos de material, las deformaciones metálicas, entre otras, y se basa en que parte de la energía que libera al ocurrir alguno de los fenómenos anteriores y se transmiten al exterior en forma de ondas acústicas, es decir, emiten sonido. En todo caso, cada máquina posee un patrón único de sonido que puede ser proporcionado por el fabricante o, en su defecto, registrarse cuando la máquina se encuentra en condiciones adecuadas de operación, y que servirá de base para que en caso de detectarse alguna desviación con respecto a dicho patrón, esto sea el indicativo de la aparición de una posible falla o mal funcionamiento. El registro de estas ondas acústicas se puede realizar mediante el uso de transductores (micrófonos) instalados en las proximidades del generador. Los transductores convierten las ondas acústicas en señales eléctricas que pueden procesarse y analizarse para realizar el diagnóstico.

La falla en la jaula del rodamiento de un generador síncrono no puede ser detectada a través de la utilización de acelerómetros, pero presenta un aumento en la emisión acústica, lo que permite la utilización de mi- crófonos para su detección, aspecto innovador en este trabajo.

\section{MARCO TEÓRICO}

\subsection{Evaluación de los micrófonos omnidireccionales}

En este trabajo se propone la utilización de micrófonos como una herramienta alternativa, segura, no invasiva y relativamente fácil de utilizar para la detección de fallas en máquinas eléctricas. Asimismo, se realiza un comparativo de los resultados obtenidos con esta metodología contra la usualmente empleada para este fin y que implica el uso de acelerómetros. Los resultados logrados resultan equiparables en cuanto al diagnóstico de fallas, ya que los micrófonos registran señales acústicas mientras que un acelerómetro, señales de vibración en la máquina misma. Es precisamente esta propuesta la contribución que se pretende mostrar en este trabajo, en el campo del diagnóstico de fallas en máquinas eléctricas.

Para esto se utilizaron micrófonos omnidireccionales Panasonic WM-61A, cuyas especificaciones son la sensitividad de -35+/$4 \mathrm{~dB}$, impedancia menor de $2,2 \mathrm{k} \Omega$, frecuencia de $20-16,000 \mathrm{~Hz}$, el voltaje máximo de operación de $10 \mathrm{~V}$, voltaje estándar de operación de $2 \mathrm{~V}$, la corriente de consumo máximo $0,5 \mathrm{~mA}$ y relación señal ruido $(S N R)>62 d B$, los cuales fueron colocados sobre bases metálicas a una distancia de $21 \mathrm{~cm}$, tanto en el eje como en el eje . 
Tratando de compensar la problemática del ruido ambiental de fondo en el área donde se encuentra trabajando la máquina por examinar, puede utilizarse un supresor de ruido para un detector de fallo Noise Cancellation Fault Detector (NCFD), que permite cancelar el ruido ambiental de fondo donde se encuentran ubicadas las máquinas eléctricas que se desean monitorear, sin afectar la emisión acústica relacionada con la falla [8].

\section{DISEÑO DE EXPERIMENTOS}

El diseño de experimentos (DDE) hace uso de técnicas y herramientas estadísticas, con la finalidad de analizar o mejorar el desempeño de un conjunto de experimentos o un experimento en particular. Tiene como propósito principal establecer conclusiones válidas y robustas acerca del proceso o sistema analizado. Puede ser empleado para establecer el grado de influencia de las variables de control o de ruido sobre una variable de respuesta observable o para establecer valores de dichas variables y mejorar u optimizar un proceso, producto o sistema hacia un valor o características establecidas como deseables.

Permite, asimismo, encontrar sin lugar a dudas aquellas variables que más aportan a la variabilidad de un proceso o experimento, y con ello generar estrategias de acción, conclusiones y niveles de operación confiables y robustos, como ya se indicó anteriormente; esto es, que sean prácticamente insensibles a la variación o ruido externo.
Tradicionalmente existen dos enfoques ligeramente distintos de la aplicación del diseño de experimentos, el que se le conoce como la metodología tradicional, y que se halla basado en la utilización de arreglos factoriales, completos o fraccionados, y la metodología Taguchi de diseño de experimentos. En este trabajo se empleó esta última para diseñar los experimentos. Debido a las características del equipo experimental construido, se seleccionaron arreglos ortogonales como los más adecuados, ya que permiten no solo probar significancia estadística de efectos principales, sino también interacciones de interés. La metodología para determinar la tabla de análisis de varianza ANDEVA (ANOVA, por sus siglas en inglés), se halla reportada a detalle en [9].

El procedimiento y las herramientas utilizados en la medición y diagnóstico de señales se encuentran reportados de manera detallada en [10].

\section{METODOLOGÍA EXPERIMENTAL}

\subsection{Ensayos y resultados}

Se llevaron a cabo ensayos con rodamientos sin falla y con grieta en la jaula de un rodamiento SKF-6303-2RSH. Las pruebas se realizaron en un banco de pruebas, que se puede observar en la Fig. 1 [10], y sus características se indican en la Tabla 1. 


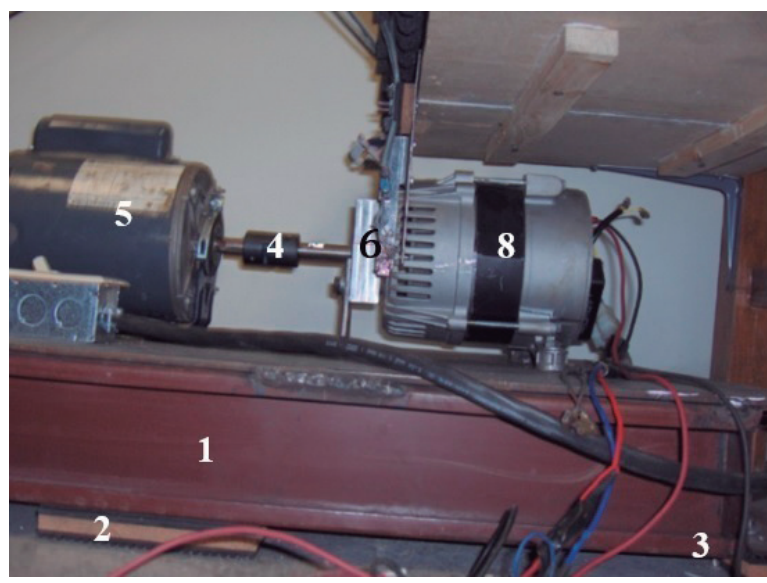

(a)

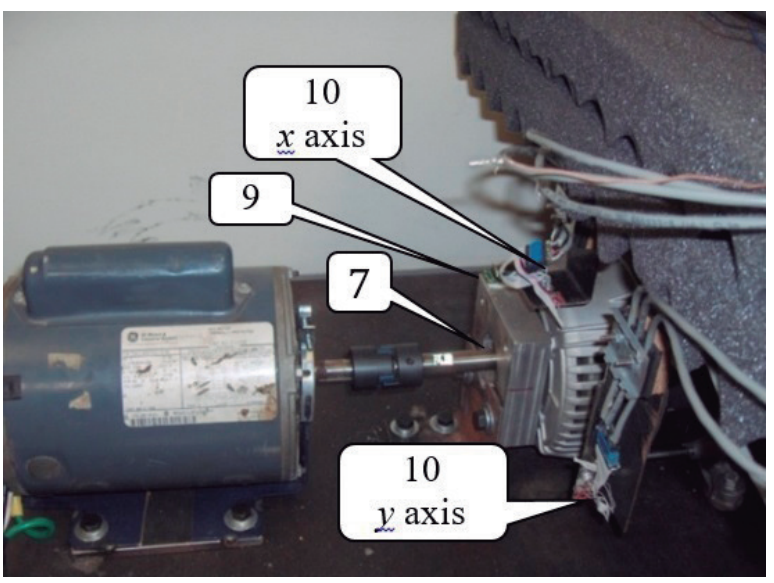

(b)

Fig. 1. Sistema mecánico: a) banco experimental, b) colocación de los transductores Fuente: elaboración propia.

Tabla 1. Características del banco de prueba

\begin{tabular}{|l|l|l|}
\hline Núm. & Cant. & \\
\hline 1 & 1 & Base (plancha de metal $6,35 \times 10^{-3} \mathrm{~m}$, polín calibre 12) \\
\hline 2 & 4 & Amortiguadores de corcho y neopreno \\
\hline 3 & 4 & Tornillos roscables \\
\hline 4 & 1 & Acoplamiento elástico lovejoy de $12,7 \times 10^{-3}$ a $12,7 \times 10^{-3} \mathrm{~m}$ \\
\hline 5 & 1 & $\begin{array}{l}\text { Motor de inducción monofásico GE, CATNO. C1158, } 1 \mathrm{Hp}, 60 \mathrm{~Hz}, 115 / 208-230 \mathrm{~V}, \\
1725 \text { rpm, } 14,7 \text { A. }\end{array}$ \\
\hline 6 & 1 & Base de aluminio de $(0,1 \times 0,1 \times 0,03 \mathrm{~m})$ \\
\hline 7 & 2 & Rodamiento SKF-6303-2RSH \\
\hline 8 & 1 & Generador síncrono, 2 Hp, 50 Vcd \\
\hline 9 & 1 & Acelerómetro 3 ejes (Analog Device EVAL-ADXL325) \\
\hline 10 & 2 & Micrófono omnidireccional (Panasonic WM-61A) \\
\hline
\end{tabular}

Fuente: elaboración propia. 


\subsection{Frecuencias de falla del} rodamiento SKF-6303-2RSH

El deterioro en alguno de los componentes del rodamiento produce picos a cierta frecuencia [10]-[16]; el valor de esta frecuencia depende de la geometría del rodamiento, así como de su velocidad de rotación, y están definidos en al menos cuatro frecuencias características que se producen en los rodamientos cuando la falla es producida en la pista exterior "(1)" (BPFO, ball pass frequency of the outer race, por sus siglas en inglés), en la pista interior "(2)" (BPFI, ball pass frequency of the inner race, por sus siglas en inglés), en las bolas o elementos rodantes "(3)" (BSF, ball spin frequency, por sus siglas en inglés), en la jaula "(4)" (FTF, fundamental train frecuency, por sus siglas en inglés), [14]-[17].

$$
B P F O=\frac{n e}{2} \cdot \frac{V_{r p m}}{60} \cdot\left[1-\frac{d}{D} \cdot \cos (\beta)\right]
$$

$$
\begin{gathered}
B P F I=\frac{n e}{2} \cdot \frac{V_{r p m}}{60} \cdot\left[1+\frac{d}{D} \cdot \cos (\beta)\right] \\
B S F=\frac{D}{d} \cdot \frac{V_{r p m}}{60} \cdot\left[1-\left(\frac{d}{D}\right)^{2} \cdot \cos ^{2}(\beta)\right] \\
F T F=\frac{N V_{r p m}}{120} \cdot\left[1-\frac{d}{D} \cdot \cos (\beta)\right]
\end{gathered}
$$

Donde $D$ es el diámetro de paso $(\mathrm{mm}), d$ el diámetro de bolas $(\mathrm{mm}), \beta$ el ángulo de contacto entre las bolas y las pistas, $V_{-} r p m$ la velocidad de rotación (rpm), y el número de elementos rodantes.

Los valores para el rodamiento SKF-6303$2 R S H$ son: $D=32 \mathrm{~mm} ; d=8,731 \mathrm{~mm} ; \beta=0^{\circ}$; ne $=7$ bolas; $V_{-} r p m=1725 \mathrm{rpm}$ (revoluciones de operación del impulsor).

Se pueden observar en la Tabla 2 los valores de las frecuencias de vibración para los elementos que componen el rodamiento.

Tabla 2. Frecuencias de falla para los elementos del rodamiento SKF-6303-2RSH

\begin{tabular}{|c|l|l|l|l|l|}
\hline $\begin{array}{c}\text { Frecuencia de } \\
\text { rotación }\end{array}$ & $\begin{array}{c}\text { Defecto en la } \\
\text { pista interior } \\
\mathrm{Hz} \text { (BPFI) }\end{array}$ & $\begin{array}{c}\text { Defectos en la } \\
\text { pista exterior } \\
\text { Hz (BPFO) }\end{array}$ & $\begin{array}{c}\text { Defectos en la } \\
\text { jaula Hz (FTF) }\end{array}$ & $\begin{array}{c}\text { Frecuencia de } \\
\text { giro de bolas } \\
\text { Hz (BSF) }\end{array}$ & $\begin{array}{c}\text { Defectos en las } \\
\text { bolas Hz }\end{array}$ \\
\hline $60(\mathrm{~Hz}) / 1725(\mathrm{rpm})$ & 128,080 & 73,170 & 10,453 & 48,764 & 97,527 \\
\hline
\end{tabular}

Fuente: elaboración propia. 
Las señales de vibración y acústicas del rodamiento fueron adquiridas a una tasa de 6000 muestras/segundo, con una tarjeta para adquisición de datos NI USB-6009.

Las señales de vibración producidas en el rodamiento de estudio fueron muestreadas a través de un sistema de adquisición que realiza la medición simultánea de la señal del acelerómetro (eje $x$ y eje $y$ ), así como con los micrófonos omnidireccionales (eje $x$ y eje $y$ ).

La Fig. 2 muestra el sistema de adquisición para los micrófonos omnidireccionales y los acelerómetros piezoeléctricos.

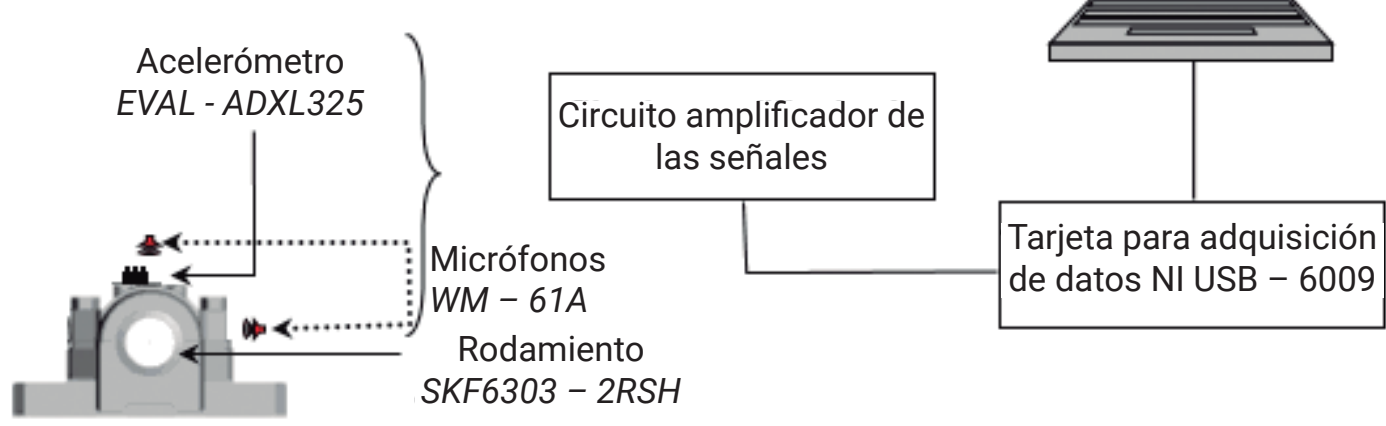

Fig. 2. Sistema de adquisición para los micrófonos y los acelerómetros piezoeléctricos Fuente: elaboración propia.

\section{METODOLOGÍA EXPERIMENTAL}

Se desarrolló un diseño experimental con las siguientes características. Se consideraron tres tipos de carga: resistiva $(R)$, inductiva $(L)$ y capacitiva $(C)$, para probar su posible influencia en la variable de respuesta, en este caso, el nivel de vibración registrado por transductores específicos para convertir las señales de los acelerómetros y de los micrófonos, respectivamente. La señal fue registrada en milivolts $(m V)$, y se seleccionaron dos niveles para cada tipo de carga; para la carga resistiva fueron de 0 y $100 \Omega$ y las cargas inductiva y capacitiva se probaron para un valor estandarizado equivalente de 0 y $100 \Omega$, lo que corresponde a 0 y 0,2667 Henrios y 26,4 $\mu$ Farad, respectivamente.

El experimento se planificó basado en un arreglo ortogonal, considerando tres tipos de carga: resistiva $(R)$, inductiva $(L)$ y capacitiva $(C)$ con dos interacciones, a dos niveles [9], [18]. La Tabla 3 muestra los factores de control y la Tabla 4, el arreglo ortogonal $L_{8}$. 
Tabla 3. Factores de control

\begin{tabular}{|l|l|l|l|}
\hline & \multicolumn{1}{|c|}{ Factores de control } & \multicolumn{1}{c|}{ Nivel 1* } & \multicolumn{1}{c|}{ Nivel 2* } \\
\hline$L$ & Inductancia & $0 \Omega$ & $100 \Omega$ \\
\hline$R$ & Resistencia & $0 \Omega$ & $100 \Omega$ \\
\hline$R L$ & Resistencia-inductancia & $0 \Omega$ & $100 \Omega$ \\
\hline$C$ & Capacitancia & $0 \Omega$ & $100 \Omega$ \\
\hline$R C$ & Resistencia-capacitancia & $0 \Omega$ & $100 \Omega$ \\
\hline
\end{tabular}

*Se reportan valores estandarizados en Ohms $(\Omega)$ en las capacitancias e inductancia para mantener unidades homogéneas.

Fuente: elaboración propia.

Se realizaron veinticuatro pruebas con tres có este diseño en los ocho casos seleccioréplicas por condición experimental. Se apli- nados que se indican en la Tabla 4.

Tabla 4. Casos de estudio para el diseño experimental

\begin{tabular}{|l|l|l|l|}
\hline $\begin{array}{c}\text { Experimento } \\
\text { No. }\end{array}$ & \multicolumn{1}{|c|}{$\begin{array}{c}\text { Condición de prueba } \\
\text { sin falla }\end{array}$} & $\begin{array}{c}\text { Experimento } \\
\text { No. }\end{array}$ & \multicolumn{1}{c|}{$\begin{array}{c}\text { Condición de prueba } \\
\text { con falla }\end{array}$} \\
\hline 1 & Acelerómetro, eje & 5 & Acelerómetro, eje \\
\hline 2 & Acelerómetro, eje & 6 & Acelerómetro, eje \\
\hline 3 & Micrófono omnidireccional, eje & 7 & Micrófono omnidireccional, eje \\
\hline 4 & Micrófono omnidireccional, eje & 8 & Micrófono omnidireccional, eje \\
\hline
\end{tabular}

Fuente: elaboración propia. 
En la Tabla 5 se calculan los valores promedio por nivel para cada factor.

Tabla 5. Arreglo ortogonal $L_{8}$

\begin{tabular}{|c|c|c|c|c|c|c|c|c|c|c|}
\hline \multirow{3}{*}{$\begin{array}{l}\frac{\pi}{0} \\
\text { อై } \\
\text { Q }\end{array}$} & \multirow{2}{*}{\multicolumn{7}{|c|}{ Factores }} & \multirow{2}{*}{\multicolumn{3}{|c|}{$\begin{array}{c}\text { Variable de respuesta ( } Y i j \\
\text { Condición de prueba }\end{array}$}} \\
\hline & & & & & & & & & & \\
\hline & L & $\mathbf{R}$ & RL & c & e & RC & e & & je $z, z$ & \\
\hline 1 & 1 & 1 & 1 & 1 & 1 & 1 & 1 & Y_11 & $Y_{-}$12 & Y_13 \\
\hline 2 & 1 & 1 & 1 & 2 & 2 & 2 & 2 & Y_21 & Y_22 & Y_23 \\
\hline 3 & 1 & 2 & 2 & 1 & 1 & 2 & 2 & Y_31 & Y_32 & Y_33 \\
\hline 4 & 1 & 2 & 2 & 2 & 2 & 1 & 1 & Y_41 & Y_42 & Y_43 \\
\hline 5 & 2 & 1 & 2 & 1 & 2 & 1 & 2 & Y_51 & Y_52 & Y_53 \\
\hline 6 & 2 & 1 & 2 & 2 & 1 & 2 & 1 & Y_61 & Y_62 & Y_63 \\
\hline 7 & 2 & 2 & 1 & 1 & 2 & 2 & 1 & Y_71 & Y_72 & Y_73 \\
\hline 8 & 2 & 2 & 1 & 2 & 1 & 1 & 2 & Y_81 & $Y_{\text {__82 }}$ & Y_83 \\
\hline
\end{tabular}

Fuente: elaboración propia.

Por tanto, se obtuvieron ocho tablas de para este fin [9], [18]. A continuación se resultados, como las mostradas en la Ta- presentan detalladamente la metodología bla 5, para cada una de las condiciones de de cálculo y el análisis de varianza. Los prueba. El análisis estadístico se realizó análisis de varianza se muestran en las Tade acuerdo con la metodología reportada blas 6 a la 15. 
Tabla 6. Diseño de ANDEVA acelerómetro eje $x$ condición sin falla

\begin{tabular}{|c|c|c|c|c|c|c|c|c|c|c|c|c|}
\hline \multirow{2}{*}{$\frac{\text { ㅇ }}{\frac{0}{2}}$} & \multicolumn{7}{|c|}{ Factores } & \multicolumn{3}{|c|}{ Acelerómetro } & \multirow[b]{2}{*}{$T i$} & \multirow[b]{2}{*}{$\mathrm{SSe}_{2}$} \\
\hline & L & $\mathbf{R}$ & RL & C & e & RC & e & \multicolumn{3}{|c|}{ Sin falla } & & \\
\hline 8 & 2 & 2 & 1 & 2 & 1 & 1 & 2 & $-3,27$ & $-4,86$ & $-4,44$ & $-12,56$ & 1,36 \\
\hline & & & & & & & & & & T.. & $-93,19$ & 5,56 \\
\hline
\end{tabular}

Fuente: elaboración propia. 
Desarrollo del análisis de varianza (ANDEVA).

a. Totales de la variable de respuesta, para cada uno de los niveles de los factores (Ta- bla 7). Las primeras 12 pruebas del arreglo se efectuaron con el factor a su nivel 1 (0

$\Omega)$ y las siguientes 12 a su nivel $2(100 \Omega)$.

$$
\begin{aligned}
& L_{1}=-4,58-4,05-3,38-3,89-5,17-4,04-3,65-4,52-3,41-2,98-3,52-3,95=-93,20 \\
& L_{2}=-3,71-4,40-3,16-3,98-3,30-3,89-3,96-3,24-3,85-3,27-4,86-4,44=-93,20 \\
& R_{1}=-4,58-4,05-3,38-3,89-5,17-4,04-3,71-4,40-3,16-3,98-3,30-3,89=-93,20 \\
& R_{2}=-3,65-4,52-3,41-2,98-3,52-3,95-3,65-4,52-3,41-2,98-3,52-3,95=-93,20 \\
& R L_{1}=-4,58-4,05-3,38-3,89-5,17-4,04-3,96-3,24-3,85-3,27-4,86-4,44=-93,20 \\
& R L_{2}=-3,65-4,52-3,41-2,98-3,52-3,95-3,71-4,40-3,16-3,98-3,30-3,89=-93,20 \\
& C_{1}=-4,58-4,05-3,38-3,65-4,52-3,41-3,71-4,40-3,16-3,96-3,24-3,85=-93,20 \\
& C_{2}=-3,89-5,17-4,04-2,98-3,52-3,95-3,98-3,30-3,89-3,27-4,86-4,44=-93,20 \\
& e_{1}=-4,58-4,05-3,38-3,65-4,52-3,41-3,98-3,30-3,89-3,27-4,86-4,44=-93,20 \\
& e_{2}=-3,89-5,17-4,04-2,98-3,52-3,95-3,71-4,40-3,16-3,96-3,24-3,85=-93,20 \\
& R C_{1}=-4,58-4,05-3,38-2,98-3,52-3,95-3,71-4,40-3,16-3,27-4,86-4,44=-93,20 \\
& R_{2}=-3,89-5,17-4,04-3,65-4,52-3,41-3,98-3,30-3,89-3,96-3,24-3,85=-93,20 \\
& e_{1}=-4,58-4,05-3,38-2,98-3,52-3,95-3,98-3,30-3,89-3,96-3,24-3,85=-93,20 \\
& e_{2}=-3,89-5,17-4,04-3,65-4,52-3,41-3,71-4,40-3,16-3,27-4,86-4,44=-93,20
\end{aligned}
$$

Tabla 7. Suma de totales para los niveles 1 y 2 de cada factor

\begin{tabular}{|l|l|l|l|l|l|l|l|}
\hline Factor & \multicolumn{1}{|c|}{$\boldsymbol{L}$} & $\boldsymbol{R}$ & $\boldsymbol{R}$ & $\boldsymbol{C}$ & $\boldsymbol{e}$ & $\boldsymbol{R}$ & $\boldsymbol{T}$ \\
\hline Nivel 1 & $-47,14$ & $-47,55$ & $-48,73$ & $-45,91$ & $-47,33$ & $-46,30$ & $-44,68$ \\
\hline Nivel 2 & $-46,06$ & $-45,65$ & $-44,47$ & $-47,29$ & $-45,87$ & $-46,90$ & $-48,52$ \\
\hline Total & $-93,20$ & $-93,20$ & $-93,20$ & $-93,20$ & $-93,20$ & $-93,20$ & $-93,20$ \\
\hline
\end{tabular}

Fuente: elaboración propia. 
b. Suma de cuadrados "(5)":

$$
\left(\operatorname{SSX}=(\text { Total Nivel } 2-\text { Total Nivel } 1)^{2} / n\right)
$$

Donde:

$n=$ representa el número total de lecturas que se tomaron $(n=24)$ :

$$
\begin{aligned}
& S S \text { de } L=\left(L_{2}-L_{1}\right)^{2} / 24=(-46,06--47,14)^{2} / 24=0,05 \text { con } 1 \mathrm{~g} . l . \\
& S S \text { de } R=\left(R_{2}-R_{1}\right)^{2} / 24=(-46,65--47,55)^{2} / 24=0,15 \text { con } 1 \mathrm{~g} . l . \\
& S S \text { de } R L=\left(R L_{2}-R L_{1}\right)^{2} / 24=(-44,47--48,73)^{2} / 24=0,76 \text { con } 1 \mathrm{~g} . l . \\
& S S \text { de } C=\left(C_{2}-C_{1}\right)^{2} / 24=(-47,29--45,91)^{2} / 24=0,08 \text { con } 1 \mathrm{~g} . l . \\
& S S \text { de } R C=\left(R C_{2}-R C_{1}\right)^{2} / 24=(-46,30--46,90)^{2} / 24=0,01 \text { con } 1 \mathrm{~g} . l . \\
& S S \text { de } e_{1}=\left(e_{2}-e_{1}\right)^{2} / 24=(-45,87--47,33)^{2} / 24=0,09 \text { con } 1 \mathrm{~g} . l . \\
& S S \text { de } e_{2}=\left(e_{2}-e_{1}\right)^{2} / 24=(-48,52--44,68)^{2} / 24=0,61 \text { con } 1 \mathrm{~g} . l .
\end{aligned}
$$

La suma de cuadrados del error (SSE) se suman "(6)".

$$
S S e=S S_{1}+S S_{2}
$$

$S S e=0,09+0,61$ con 2 g.l

c. Cálculo de, se obtiene dividiendo la suma de cuadrados (SS) entre los grados de libertad (g.l) "(7)". 


$$
M S=S S L / g . l
$$

$$
\begin{aligned}
& M S \text { de } L=S S L / g . l .=0,05 / 1=0,05 \\
& M S \text { de } R=S S R / g . l .=0,15 / 1=0,15 \\
& M S \text { de } R L=S S R L / g . l .=0,76 / 1=0,76 \\
& M S \text { de } C=S S C / g . l .=0,08 / 1=0,08 \\
& M S \text { de } R C=S S R C / g . l .=0,01 / 1=0,01 \\
& M S \text { de } e_{1}=S S e_{1} / g . l .=0,69 / 2=0,35 \\
& M S \text { de } e_{2}=S S e_{2} / g . l .=5,56 / 16=0,35
\end{aligned}
$$

F_exp, se obtiene de dividir el valor de de cada factor, entre el valor de para la estimación del error "(8)".

$$
\begin{gathered}
F_{\text {exp }}=M S / M S\left(e_{2}\right) \\
F_{\text {exp }} \text { de } L=M S(L) / M S\left(e_{2}\right)=0,05 / 0,35=0,15 \\
F_{\text {exp }} \text { de } R=M S(R) / M S\left(e_{2}\right)=0,15 / 0,35=0,44 \\
F_{\text {exp }} \text { de } R L=M S(R L) / M S\left(e_{2}\right)=0,76 / 0,35=2,20
\end{gathered}
$$




$$
\begin{aligned}
& F_{\text {exp }} \text { de } C=M S(C) / M S\left(e_{2}\right)=0,08 / 0,35=0,23 \\
& F_{\text {exp }} \text { de } R C=M S(R C) / M S\left(e_{2}\right)=0,01 / 0,35=0,04
\end{aligned}
$$

d. Se construye la tabla ANDEVA (Tabla 8):

Todos aquellos factores que tienen un valor de mayor que 4,41 se considera que afectan la variable de respuesta. Estos son llamados factores significantes.

\begin{tabular}{|c|c|c|c|c|c|}
\hline Factor & ss & g.l. & MS & $F_{\text {exp }}$ & \\
\hline$L$ & 0,05 & 1 & 0,05 & 0,15 & \multirow{9}{*}{$\begin{array}{l}\text { Se probarán contra } \\
F_{0,05,1,18}=4,41\end{array}$} \\
\hline$R$ & 0,15 & 1 & 0,15 & 0,44 & \\
\hline$R L$ & 0,76 & 1 & 0,76 & 2,20 & \\
\hline$c$ & 0,08 & 1 & 0,08 & 0,23 & \\
\hline$R C$ & 0,01 & 1 & 0,01 & 0,04 & \\
\hline$e_{1}$ & 0,69 & 2 & 0,35 & -- & \\
\hline$e_{2}$ & 5,56 & 16 & 0,35 & -- & \\
\hline Total & 7,31 & 23 & & & \\
\hline$e$ & 6,25 & 18 & 0,35 & & \\
\hline
\end{tabular}

Tabla 8. Construcción de ANDEVA

Fuente: elaboración propia.

$$
\frac{M S_{e_{1}}}{M S_{e_{2}}}=\frac{0,35}{0,35}=1,00
$$

El error primario $e_{1}<e_{2}$ el error secundario. Se prueba entonces contra $e$. 
Ciencia e Ingeniería Neogranadina

Tabla 9. Diseño de ANDEVA acelerómetro eje condición sin falla.

\begin{tabular}{|c|c|c|c|c|c|c|c|c|c|c|c|c|}
\hline \multirow{2}{*}{$\begin{array}{l}\frac{0}{0} \\
\frac{0}{2} \\
\frac{2}{2}\end{array}$} & \multicolumn{7}{|c|}{ Factores } & \multicolumn{4}{|c|}{ Acelerómetro } & \multirow[b]{2}{*}{$\mathrm{SSe}_{2}$} \\
\hline & & & & & & & & \multicolumn{3}{|c|}{ Sin falla } & Ti & \\
\hline 1 & 1 & 1 & 1 & 1 & 1 & 1 & 1 & $-6,51$ & $-6,79$ & $-6,58$ & $-19,88$ & 0,04 \\
\hline 2 & 1 & 1 & 1 & 2 & 2 & 2 & 2 & $-6,29$ & $-6,53$ & $-6,15$ & $-18,97$ & 0,07 \\
\hline 3 & 1 & 2 & 2 & 1 & 1 & 2 & 2 & $-5,76$ & $-6,42$ & $-6,24$ & $-18,52$ & 0,26 \\
\hline 4 & 1 & 2 & 2 & 2 & 2 & 1 & 1 & $-6,31$ & $-5,67$ & $-6,43$ & $-18,42$ & 0,33 \\
\hline 5 & 2 & 1 & 2 & 1 & 2 & 1 & 2 & $-5,83$ & $-6,46$ & $-6,29$ & $-18,57$ & 0,21 \\
\hline 6 & 2 & 1 & 2 & 2 & 1 & 2 & 1 & $-6,46$ & $-5,55$ & $-5,90$ & $-17,92$ & 0,42 \\
\hline 7 & 2 & 2 & 1 & 1 & 2 & 2 & 1 & $-5,89$ & $-6,29$ & $-6,28$ & $-18,46$ & 0,11 \\
\hline 8 & 2 & 2 & 1 & 2 & 1 & 1 & 2 & $-6,07$ & $-6,72$ & $-6,80$ & $-19,59$ & 0,31 \\
\hline & & & & & & & & & & $\mathrm{T}$. . & $-150,32$ & 1,76 \\
\hline
\end{tabular}




\begin{tabular}{|c|c|c|c|c|c|}
\hline Factor & sS & g.l. & MS & $F_{\text {exp }}$ & \\
\hline$L$ & 0,06 & 1 & 0,06 & 0,62 & \multirow{9}{*}{$\begin{array}{l}\text { Se probarán contra } \\
F_{0,05,1,18}=4,41\end{array}$} \\
\hline$R$ & 0,00 & 1 & 0,10 & 0,05 & \\
\hline$R L$ & 0,50 & 1 & 0,50 & 4,79 & \\
\hline$C$ & 0,01 & 1 & 0,01 & 0,12 & \\
\hline$R C$ & 0,28 & 1 & 0,28 & 2,67 & \\
\hline$e_{1}$ & 0,13 & 2 & 0,07 & -- & \\
\hline$e_{2}$ & 1,76 & 16 & 0,11 & -- & \\
\hline Total & 2,75 & 23 & & & \\
\hline e & 1,89 & 18 & 0,10 & & \\
\hline
\end{tabular}

Fuente: elaboración propia.

$$
\frac{M S_{e_{1}}}{M S_{e_{2}}}=\frac{0,07}{0,11}=0,60
$$

El error primario $e_{1}<e_{2}$ el error secundario. Se prueba entonces contra $e$.

Tabla 10. Diseño de ANDEVA micrófono omnidireccional eje condición sin falla.

\begin{tabular}{|c|c|c|c|c|c|c|c|c|c|c|c|c|}
\hline \multirow{3}{*}{ ֻٓ } & \multicolumn{7}{|c|}{ Factores } & \multicolumn{3}{|c|}{ Micrófono omnidireccional } & \multirow{3}{*}{$\mathrm{Ti}$} & \multirow{3}{*}{$\mathrm{SSe}_{2}$} \\
\hline & \multirow{2}{*}{$\mathbf{L}$} & \multirow{2}{*}{$\mathbf{R}$} & \multirow{2}{*}{ RL } & \multirow{2}{*}{ C } & \multirow{2}{*}{ e } & \multirow{2}{*}{ RC } & \multirow{2}{*}{ e } & \multicolumn{3}{|c|}{ Sin falla } & & \\
\hline & & & & & & & & & Eje $x$ & & & \\
\hline 1 & 1 & 1 & 1 & 1 & 1 & 1 & 1 & $-13,30$ & $-9,01$ & $-9,09$ & $-31,40$ & 12,04 \\
\hline 2 & 1 & 1 & 1 & 2 & 2 & 2 & 2 & $-12,75$ & $-3,89$ & $-10,27$ & $-26,92$ & 41,80 \\
\hline 3 & 1 & 2 & 2 & 1 & 1 & 2 & 2 & $-9,64$ & $-8,95$ & $-2,52$ & $-21,11$ & 30,83 \\
\hline
\end{tabular}


Ciencia e Ingeniería Neogranadina

\begin{tabular}{|c|c|c|c|c|c|c|c|c|c|c|c|c|}
\hline \multirow{4}{*}{ 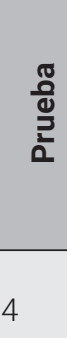 } & \multicolumn{7}{|c|}{ Factores } & \multicolumn{3}{|c|}{ Micrófono omnidireccional } & \multirow{3}{*}{ Ti } & \multirow{3}{*}{$\mathrm{SSe}_{2}$} \\
\hline & \multirow{2}{*}{$\mathbf{L}$} & \multirow{2}{*}{$\mathbf{R}$} & \multirow{2}{*}{$\mathbf{R L}$} & \multirow{2}{*}{ C } & \multirow{2}{*}{ e } & \multirow{2}{*}{ RC } & \multirow{2}{*}{ e } & \multicolumn{3}{|c|}{ Sin falla } & & \\
\hline & & & & & & & & & Eje $x$ & & & \\
\hline & 1 & 2 & 2 & 2 & 2 & 1 & 1 & $-1,75$ & $-7,52$ & $-7,65$ & $-16,93$ & 22,70 \\
\hline 5 & 2 & 1 & 2 & 1 & 2 & 1 & 2 & $-6,61$ & $-12,41$ & $-1,67$ & $-20,69$ & 57,81 \\
\hline 6 & 2 & 1 & 2 & 2 & 1 & 2 & 1 & $-4,10$ & $-5,42$ & $-13,34$ & $-22,87$ & 49,93 \\
\hline 7 & 2 & 2 & 1 & 1 & 2 & 2 & 1 & $-16,46$ & $-0,35$ & $-14,10$ & $-30,92$ & 151,36 \\
\hline 8 & 2 & 2 & 1 & 2 & 1 & 1 & 2 & $-6,14$ & $-11,43$ & $-15,81$ & $-33,38$ & 46,93 \\
\hline & & & & & & & & & & T.. & $-204,22$ & 413,40 \\
\hline
\end{tabular}

\begin{tabular}{|c|c|c|c|c|c|}
\hline Factor & $s s$ & g.l. & MS & $F_{\text {exp }}$ & \\
\hline$L$ & 5,51 & 1 & 5,51 & 0,24 & \multirow{9}{*}{$\begin{array}{l}\text { Se probarán contra } \\
F_{0,05,1,18}=4,41\end{array}$} \\
\hline$R$ & 0,01 & 1 & 0,01 & 0,00 & \\
\hline$R L$ & 70,09 & 1 & 70,09 & 3,00 & \\
\hline$c$ & 0,68 & 1 & 0,68 & 0,03 & \\
\hline$R C$ & 0,01 & 1 & 0,01 & 0,00 & \\
\hline$e_{1}$ & 7,37 & 2 & 3,68 & -- & \\
\hline$e_{2}$ & 413,40 & 16 & 25,84 & -- & \\
\hline Total & 497,06 & 23 & & & \\
\hline$e$ & 420,76 & 18 & 23,38 & & \\
\hline
\end{tabular}

Fuente: elaboración propia. 


$$
\frac{M S_{e_{1}}}{M S_{e_{2}}}=\frac{3,68}{25,84}=0,14
$$

El error primario $e_{1}<e_{2}$, el error secundario. Se prueba entonces contra $e$.

Tabla 11. Diseño de ANDEVA micrófono omnidireccional eje condición sin falla

\begin{tabular}{|c|c|c|c|c|c|c|c|c|c|c|c|c|}
\hline \multirow{4}{*}{ 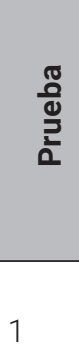 } & \multicolumn{7}{|c|}{ Factores } & \multirow{2}{*}{\multicolumn{3}{|c|}{$\begin{array}{c}\text { Micrófono omnidireccional } \\
\text { Sin falla }\end{array}$}} & \multirow{3}{*}{$T i$} & \multirow{3}{*}{$\mathrm{SSe}_{2}$} \\
\hline & \multirow{2}{*}{ L } & \multirow{2}{*}{$\mathbf{R}$} & \multirow{2}{*}{ RL } & \multirow{2}{*}{ C } & \multirow{2}{*}{ e } & \multirow{2}{*}{ RC } & \multirow{2}{*}{ e } & & & & & \\
\hline & & & & & & & & \multicolumn{3}{|c|}{ Eje y } & & \\
\hline & 1 & 1 & 1 & 1 & 1 & 1 & 1 & $-4,55$ & $-2,03$ & 2,09 & $-4,48$ & 22,43 \\
\hline 2 & 1 & 1 & 1 & 2 & 2 & 2 & 2 & $-4,41$ & $-3,37$ & $-3,20$ & $-10,98$ & 0,85 \\
\hline 3 & 1 & 2 & 2 & 1 & 1 & 2 & 2 & $-3,04$ & $-4,57$ & $-0,69$ & $-8,29$ & 7,64 \\
\hline 4 & 1 & 2 & 2 & 2 & 2 & 1 & 1 & $-2,09$ & $-2,06$ & $-7,15$ & $-11,30$ & 17,18 \\
\hline 5 & 2 & 1 & 2 & 1 & 2 & 1 & 2 & $-1,52$ & $-8,74$ & $-0,01$ & $-10,27$ & 43,49 \\
\hline 6 & 2 & 1 & 2 & 2 & 1 & 2 & 1 & $-1,43$ & $-1,44$ & $-5,03$ & $-7,91$ & 8,60 \\
\hline 7 & 2 & 2 & 1 & 1 & 2 & 2 & 1 & $-7,71$ & $-1,50$ & $-3,96$ & $-13,17$ & 19,56 \\
\hline 8 & 2 & 2 & 1 & 2 & 1 & 1 & 2 & 2,19 & $-4,22$ & $-2,87$ & $-4,90$ & 22,85 \\
\hline & & & & & & & & & & T.. & $-71,30$ & 142,60 \\
\hline
\end{tabular}


Ciencia e Ingeniería Neogranadina

\begin{tabular}{|c|c|c|c|c|c|}
\hline Factor & ss & g.l. & MS & $F_{\text {exp }}$ & \\
\hline$L$ & 0,06 & 1 & 0,06 & 0,01 & \multirow{9}{*}{$\begin{array}{l}\text { Se probarán contra } \\
F_{0,05,1,18}=4,41\end{array}$} \\
\hline$R$ & 0,68 & 1 & 0,67 & 0,08 & \\
\hline$R L$ & 0,75 & 1 & 0,75 & 0,08 & \\
\hline$C$ & 0,05 & 1 & 0,05 & 0,01 & \\
\hline$R C$ & 3,68 & 1 & 3,68 & 0,41 & \\
\hline$e_{1}$ & 17,11 & 2 & 8,56 & -- & \\
\hline$e_{2}$ & 142,60 & 16 & 8,91 & -- & \\
\hline Total & 164,93 & 23 & & & \\
\hline e & 159,72 & 18 & 8,87 & & \\
\hline
\end{tabular}

Fuente: elaboración propia.

$$
\frac{M S_{e_{1}}}{M S_{e_{2}}}=\frac{8,56}{8,91}=0,96
$$

El error primario $e_{1}<e_{2^{\prime}}$ el error secundario. Se prueba entonces contra $e$.

Tabla 12. Diseño de ANDEVA acelerómetro eje $x$ condición con falla

\begin{tabular}{|c|c|c|c|c|c|c|c|c|c|c|c|c|}
\hline \multirow{3}{*}{$\begin{array}{l}\frac{\pi}{0} \\
\frac{0}{2} \\
\frac{2}{2}\end{array}$} & \multicolumn{7}{|c|}{ Factores } & \multicolumn{3}{|c|}{ Acelerómetro } & \multirow{3}{*}{ Ti } & \multirow{3}{*}{$\mathrm{SSe}_{2}$} \\
\hline & \multirow{2}{*}{$\mathbf{L}$} & \multirow{2}{*}{$\mathbf{R}$} & \multirow{2}{*}{ RL } & \multirow{2}{*}{ C } & \multirow{2}{*}{ e } & \multirow{2}{*}{ RC } & \multirow{2}{*}{ e } & \multicolumn{3}{|c|}{ Con falla } & & \\
\hline & & & & & & & & & Eje $x$ & & & \\
\hline 1 & 1 & 1 & 1 & 1 & 1 & 1 & 1 & 36,56 & 36,47 & 34,14 & 107,16 & 3,77 \\
\hline 2 & 1 & 1 & 1 & 2 & 2 & 2 & 2 & 38,45 & 38,17 & 37,98 & 114,59 & 0,11 \\
\hline 3 & 1 & 2 & 2 & 1 & 1 & 2 & 2 & 36,81 & 35,45 & 36,17 & 108,43 & 0,91 \\
\hline
\end{tabular}




\begin{tabular}{|c|c|c|c|c|c|c|c|c|c|c|c|c|}
\hline \multirow{4}{*}{ 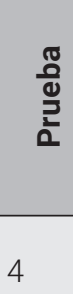 } & \multicolumn{7}{|c|}{ Factores } & \multirow{2}{*}{\multicolumn{3}{|c|}{$\begin{array}{c}\text { Acelerómetro } \\
\text { Con falla }\end{array}$}} & \multirow{3}{*}{$\mathrm{Ti}$} & \multirow{3}{*}{$\mathrm{SSe}_{2}$} \\
\hline & \multirow{2}{*}{$\mathbf{L}$} & \multirow{2}{*}{$\mathbf{R}$} & \multirow{2}{*}{$\mathbf{R L}$} & \multirow{2}{*}{ C } & \multirow{2}{*}{ e } & \multirow{2}{*}{ RC } & \multirow{2}{*}{ e } & & & & & \\
\hline & & & & & & & & \multicolumn{3}{|c|}{ Eje $x$} & & \\
\hline & 1 & 2 & 2 & 2 & 2 & 1 & 1 & 38,08 & 38,08 & 37,71 & 113,88 & 0,09 \\
\hline 5 & 2 & 1 & 2 & 1 & 2 & 1 & 2 & 37,55 & 38,27 & 37,86 & 113,68 & 0,26 \\
\hline 6 & 2 & 1 & 2 & 2 & 1 & 2 & 1 & 37,65 & 37,42 & 38,16 & 113,24 & 0,29 \\
\hline 7 & 2 & 2 & 1 & 1 & 2 & 2 & 1 & 37,39 & 38,05 & 38,12 & 113,56 & 0,32 \\
\hline 8 & 2 & 2 & 1 & 2 & 1 & 1 & 2 & 33,69 & 32,50 & 33,41 & 99,60 & 0,78 \\
\hline & & & & & & & & & & T.. & 884,14 & 6,53 \\
\hline
\end{tabular}

\begin{tabular}{|c|c|c|c|c|c|}
\hline Factor & ss & g.l. & MS & $F_{\text {exp }}$ & \\
\hline$L$ & 0,66 & 1 & 0,66 & 0,04 & \multirow{9}{*}{$\begin{array}{l}\text { Se probarán contra } \\
F_{0,05,1,2}=18,51\end{array}$} \\
\hline$R$ & 7,26 & 1 & 7,26 & 0,40 & \\
\hline$R L$ & 8,53 & 1 & 8,53 & 0,47 & \\
\hline$C$ & 0,09 & 1 & 0,09 & 0,01 & \\
\hline$R C$ & 10,00 & 1 & 10,00 & 0,55 & \\
\hline$e_{1}$ & 36,55 & 2 & 18,28 & 1,00 & \\
\hline$e_{2}$ & 6,53 & 16 & 0,41 & 0,02 & \\
\hline Total & 69,63 & 23 & & & \\
\hline$e$ & 159,72 & 18 & 8,87 & & \\
\hline
\end{tabular}

Fuente: elaboración propia. 


$$
\frac{M S_{e_{1}}}{M S_{e_{2}}}=\frac{18,28}{0,41}=44,79
$$

El error primario $e_{1}>e_{2}$ el error secundario. Se prueba entonces contra $e_{1}$.

Tabla 13. Diseño de ANDEVA acelerómetro eje condición con falla

\begin{tabular}{|c|c|c|c|c|c|c|c|c|c|c|c|c|}
\hline \multirow{2}{*}{ 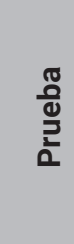 } & \multicolumn{7}{|c|}{ Factores } & \multicolumn{3}{|c|}{ Acelerómetro } & \multirow[b]{2}{*}{ Ti } & \multirow[b]{2}{*}{$\mathrm{SSe}_{2}$} \\
\hline & $\mathbf{L}$ & $\mathbf{R}$ & RL & C & e & $\mathrm{RC}$ & e & \multicolumn{3}{|c|}{ Con falla } & & \\
\hline 8 & 2 & 2 & 1 & 2 & 1 & 1 & 2 & 31,31 & 31,89 & 31,69 & 94,91 & 0,16 \\
\hline & & & & & & & & & & T.. & 837,73 & 2,64 \\
\hline
\end{tabular}




\begin{tabular}{|c|c|c|c|c|c|}
\hline Factor & ss & g.l. & MS & $F_{\text {exp }}$ & \\
\hline$L$ & 2,42 & 1 & 2,42 & 0,56 & \multirow{8}{*}{$\begin{array}{l}\text { Se probarán contra } \\
F_{0,05,1,2}=18,51\end{array}$} \\
\hline$R$ & 0,84 & 1 & 0,84 & 0,19 & \\
\hline$R L$ & 14,69 & 1 & 14,69 & 3,38 & \\
\hline$C$ & 4,94 & 1 & 4,94 & 1,14 & \\
\hline$R C$ & 11,74 & 1 & 11,74 & 2,70 & \\
\hline$e_{1}$ & 8,70 & 2 & 4,35 & 1,00 & \\
\hline$e_{2}$ & 2,64 & 16 & 0,16 & 0,04 & \\
\hline Total & 45,97 & 23 & & & \\
\hline
\end{tabular}

Fuente: elaboración propia.

$$
\frac{M S_{e_{1}}}{M S_{e_{2}}}=\frac{4,35}{0,16}=26,38
$$

El error primario $e_{1}>e_{2}$ el error secundario. Se prueba entonces contra $e_{1}$.

Tabla 14. Diseño de ANDEVA micrófono omnidireccional eje $x$ condición con falla

\begin{tabular}{|c|c|c|c|c|c|c|c|c|c|c|c|c|}
\hline \multirow{3}{*}{ 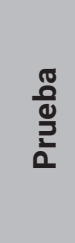 } & \multicolumn{7}{|c|}{ Factores } & \multicolumn{3}{|c|}{ Micrófono omnidireccional } & \multirow{3}{*}{$T i$} & \multirow{3}{*}{$\mathrm{SSe}_{2}$} \\
\hline & \multirow{2}{*}{$\mathbf{L}$} & \multirow{2}{*}{$\mathbf{R}$} & \multirow{2}{*}{ RL } & \multirow{2}{*}{ C } & \multirow{2}{*}{ e } & \multirow{2}{*}{ RC } & \multirow{2}{*}{ e } & \multicolumn{3}{|c|}{ Con falla } & & \\
\hline & & & & & & & & & Eje $x$ & & & \\
\hline 1 & 1 & 1 & 1 & 1 & 1 & 1 & 1 & $-6,04$ & 5,07 & 6,29 & 5,31 & 92,39 \\
\hline 2 & 1 & 1 & 1 & 2 & 2 & 2 & 2 & $-1,36$ & $-7,44$ & $-4,50$ & $-13,30$ & 18,50 \\
\hline
\end{tabular}


Ciencia e Ingeniería Neogranadina

\begin{tabular}{|c|c|c|c|c|c|c|c|c|c|c|c|c|}
\hline \multirow{4}{*}{$\begin{array}{l}\frac{\pi}{0} \\
\frac{\pi}{2} \\
3\end{array}$} & \multicolumn{7}{|c|}{ Factores } & \multirow{2}{*}{\multicolumn{3}{|c|}{$\begin{array}{c}\text { Micrófono omnidireccional } \\
\text { Con falla }\end{array}$}} & \multirow{3}{*}{$T i$} & \multirow{3}{*}{$\mathrm{SSe}_{2}$} \\
\hline & \multirow{2}{*}{ L } & \multirow{2}{*}{$\mathbf{R}$} & \multirow{2}{*}{ RL } & \multirow{2}{*}{ C } & \multirow{2}{*}{ e } & \multirow{2}{*}{ RC } & \multirow{2}{*}{ e } & & & & & \\
\hline & & & & & & & & \multicolumn{3}{|c|}{ Eje $x$} & & \\
\hline & 1 & 2 & 2 & 1 & 1 & 2 & 2 & $-6,56$ & $\mid-11,96$ & $-2,64$ & $-21,17$ & 43,81 \\
\hline 4 & 1 & 2 & 2 & 2 & 2 & 1 & 1 & $-12,92$ & $-3,79$ & $-12,15$ & $-28,86$ & 51,30 \\
\hline 5 & 2 & 1 & 2 & 1 & 2 & 1 & 2 & 0,04 & 3,09 & $-9,67$ & $-6,55$ & 88,89 \\
\hline 6 & 2 & 1 & 2 & 2 & 1 & 2 & 1 & $-7,55$ & $-3,14$ & 6,75 & $-3,93$ & 107,27 \\
\hline 7 & 2 & 2 & 1 & 1 & 2 & 2 & 1 & $-2,92$ & $-3,30$ & 13,21 & 6,98 & 177,64 \\
\hline 8 & 2 & 2 & 1 & 2 & 1 & 1 & 2 & $-7,37$ & $-6,36$ & $-3,07$ & $-16,80$ & 10,09 \\
\hline & & & & & & & & & & T.. & $-78,31$ & 589,89 \\
\hline
\end{tabular}

\begin{tabular}{|c|c|c|c|c|c|}
\hline Factor & ss & g.l. & MS & $F_{\text {exp }}$ & \\
\hline$L$ & 59,28 & 1 & 59,28 & 1,64 & \multirow{9}{*}{$\begin{array}{l}\text { Se probarán contra } \\
F_{0,05,1,18}=4,41\end{array}$} \\
\hline$R$ & 71,34 & 1 & 71,34 & 1,98 & \\
\hline$R L$ & 76,00 & 1 & 76,00 & 2,11 & \\
\hline c & 93,95 & 1 & 93,95 & 2,61 & \\
\hline$R C$ & 9,98 & 1 & 9,98 & 0,28 & \\
\hline$e_{1}$ & 59,10 & 2 & 29,55 & -- & \\
\hline$e_{2}$ & 589,89 & 16 & 36,87 & -- & \\
\hline Total & 164,93 & 23 & & & \\
\hline e & 648,99 & 18 & 36,06 & & \\
\hline
\end{tabular}

Fuente: elaboración propia. 


$$
\frac{M S_{e_{1}}}{M S_{e_{2}}}=\frac{29,55}{36,87}=0,80
$$

El error primario $e_{1}<e_{2}$ el error secundario. Se prueba entonces contra $e$.

Tabla 15. Diseño de ANDEVA micrófono omnidireccional eje y condición con falla

\begin{tabular}{|c|c|c|c|c|c|c|c|c|c|c|c|c|}
\hline \multirow{4}{*}{$\begin{array}{l}\frac{\pi}{0} \\
\stackrel{0}{2} \\
\text { 릉 }\end{array}$} & \multicolumn{7}{|c|}{ Factores } & \multirow{2}{*}{\multicolumn{3}{|c|}{$\begin{array}{c}\text { Micrófono omnidireccional } \\
\text { Con falla }\end{array}$}} & \multirow{3}{*}{$T i$} & \multirow{3}{*}{ SSe } \\
\hline & \multirow{2}{*}{ L } & \multirow{2}{*}{$\mathbf{R}$} & \multirow{2}{*}{ RL } & \multirow{2}{*}{ C } & \multirow{2}{*}{ e } & \multirow{2}{*}{ RC } & \multirow{2}{*}{ e } & & & & & \\
\hline & & & & & & & & \multicolumn{3}{|c|}{ Eje $y$} & & \\
\hline & 1 & 1 & 1 & 1 & 1 & 1 & 1 & 1,91 & 5,68 & \begin{tabular}{|l|}
4,07 \\
\end{tabular} & 11,66 & 7,17 \\
\hline 2 & 1 & 1 & 1 & 2 & 2 & 2 & 2 & $-2,22$ & 2,34 & $\mid-1,20$ & $-1,08$ & 11,46 \\
\hline 3 & 1 & 2 & 2 & 1 & 1 & 2 & 2 & $-1,42$ & $\mid-0,94$ & $-1,47$ & $-3,83$ & 0,17 \\
\hline 4 & 1 & 2 & 2 & 2 & 2 & 1 & 1 & $-0,34$ & $\mid-5,63$ & $\mid-7,07$ & $-13,04$ & 25,14 \\
\hline 5 & 2 & 1 & 2 & 1 & 2 & 1 & 2 & $-5,00$ & 4,28 & $-1,53$ & $-2,24$ & 43,94 \\
\hline 6 & 2 & 1 & 2 & 2 & 1 & 2 & 1 & $-2,27$ & $-7,02$ & $-0,98$ & $-10,26$ & 20,23 \\
\hline 7 & 2 & 2 & 1 & 1 & 2 & 2 & 1 & $-6,60$ & 3,76 & 0,46 & $-2,38$ & 56,01 \\
\hline 8 & 2 & 2 & 1 & 2 & 1 & 1 & 2 & $-0,35$ & $-1,59$ & 2,25 & 0,30 & 7,66 \\
\hline & & & & & & & & & & T.. & $-20,88$ & 171,78 \\
\hline
\end{tabular}




\begin{tabular}{|c|c|c|c|c|c|}
\hline Factor & ss & g.l. & MS & $F_{\text {exp }}$ & \\
\hline$L$ & 2,86 & 1 & 2,85 & 0,28 & \multirow{9}{*}{$\begin{array}{l}\text { Se probarán contra } \\
F_{0,05,1,18}=4,41\end{array}$} \\
\hline$R$ & 12,08 & 1 & 12,08 & 1,17 & \\
\hline$R L$ & 59,79 & 1 & 59,79 & 5,80 & \\
\hline C & 31,02 & 1 & 31,02 & 3,01 & \\
\hline$R C$ & 8,45 & 1 & 8,45 & 0,82 & \\
\hline$e_{1}$ & 13,65 & 2 & 6,82 & -- & \\
\hline$e_{2}$ & 171,78 & 16 & 10,74 & -- & \\
\hline Total & 299,64 & 23 & & & \\
\hline e & 185,43 & 18 & 10,30 & & \\
\hline
\end{tabular}

Fuente: elaboración propia.

$$
\frac{M S_{e_{1}}}{M S_{e_{2}}}=\frac{6,82}{10,74}=0,64
$$

El error primario $e_{1}<e_{2}$ el error secundario. Se prueba entonces contra $e$.

En la Fig. 3 se muestran los resultados obtenidos para la señal horizontal de vibración del generador sin falla graficada en amplitud versus frecuencia. Entretanto, en la Fig. 4 se presentan los resultados de la señal horizontal de vibración del generador con falla graficada en amplitud versus frecuencia.
En la Fig. 5 se muestran los resultados obtenidos para la señal horizontal acústica del generador sin falla graficada en amplitud versus frecuencia del micrófono omnidireccional. La Fig. 6 presenta los resultados de la señal horizontal acústica del generador con falla graficada en amplitud versus frecuencia del micrófono omnidireccional. 


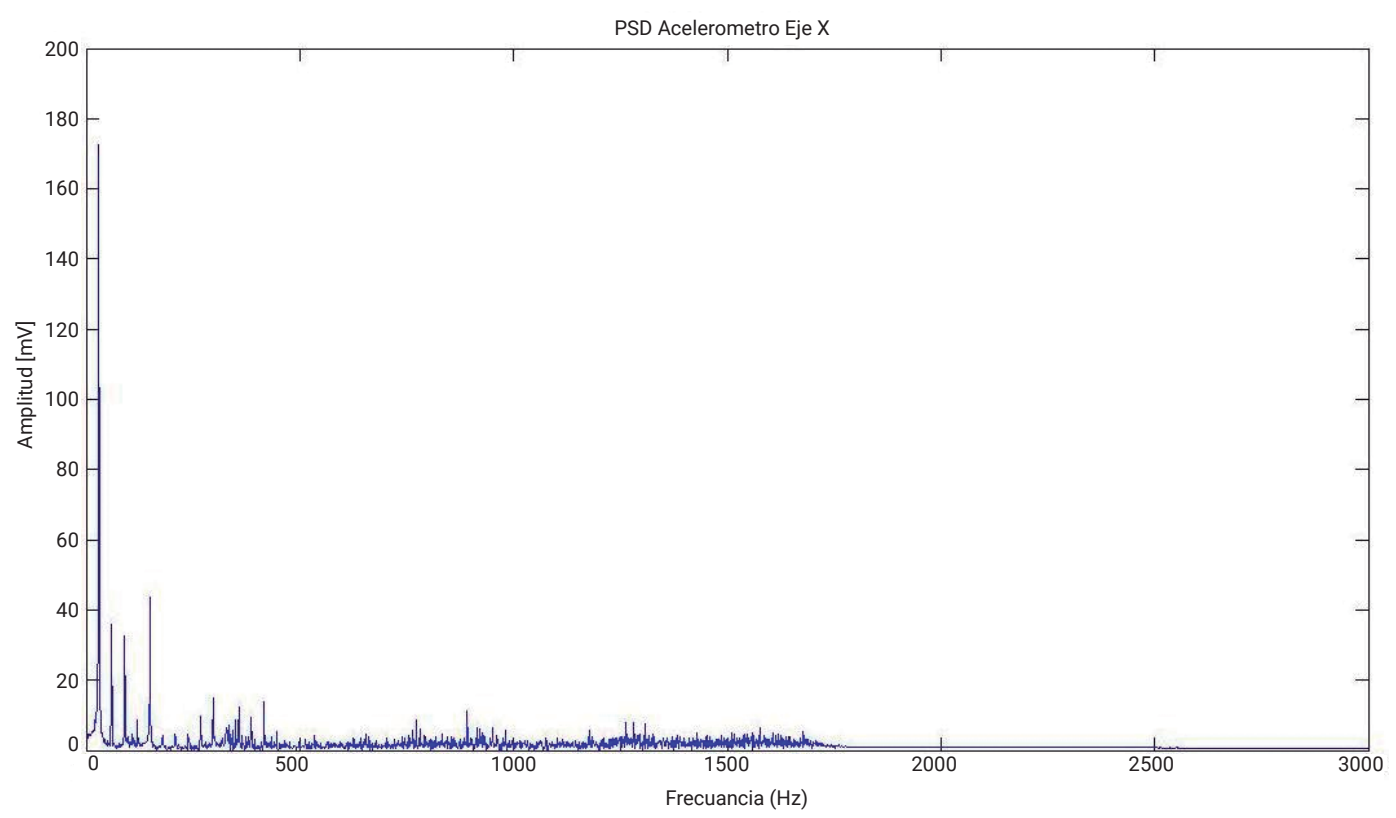

Fig. 3. Señal de vibración horizontal del acelerómetro para la condición sin falla Fuente: elaboración propia.

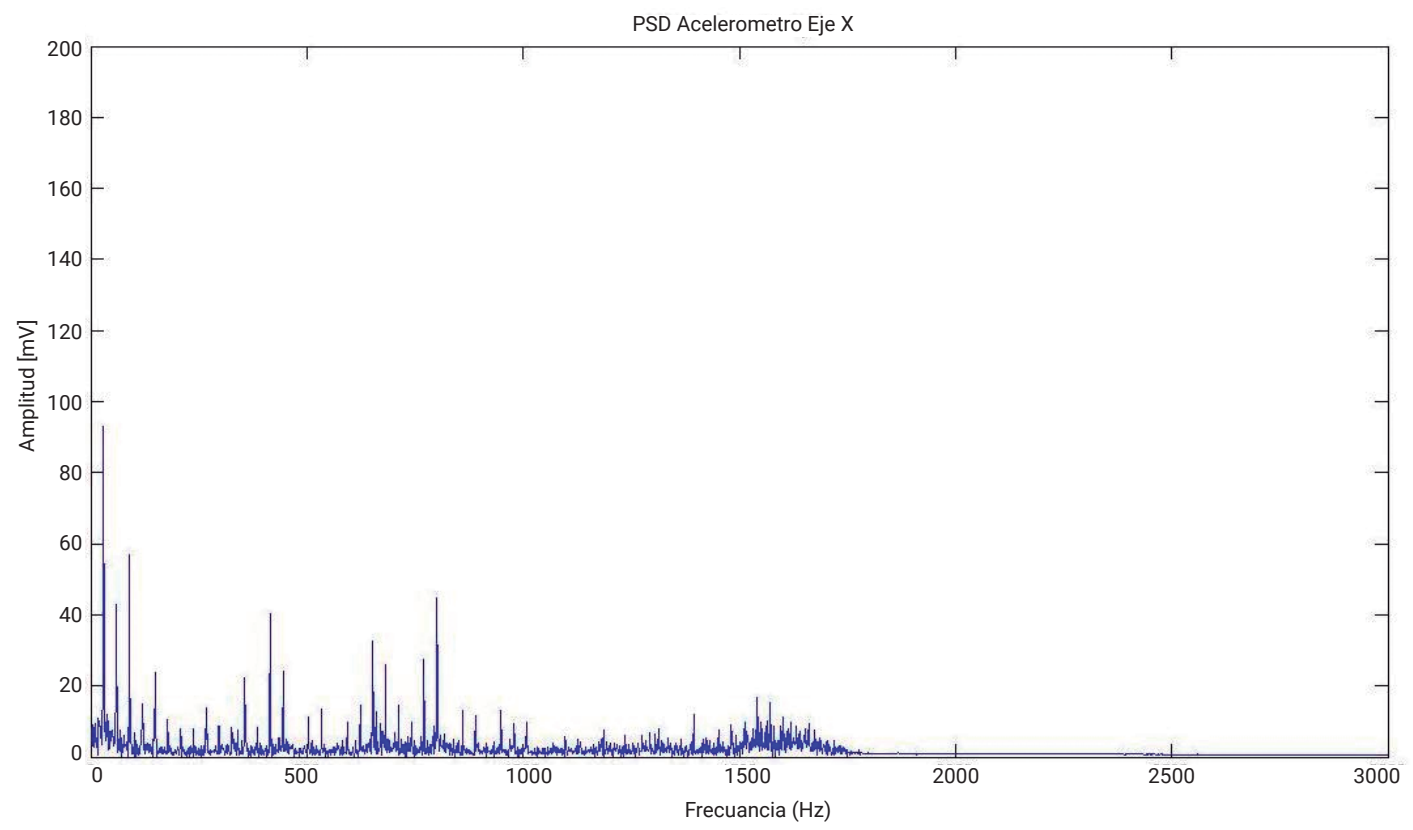

Fig. 4. Señal de vibración horizontal del acelerómetro para la condición con falla Fuente: elaboración propia. 


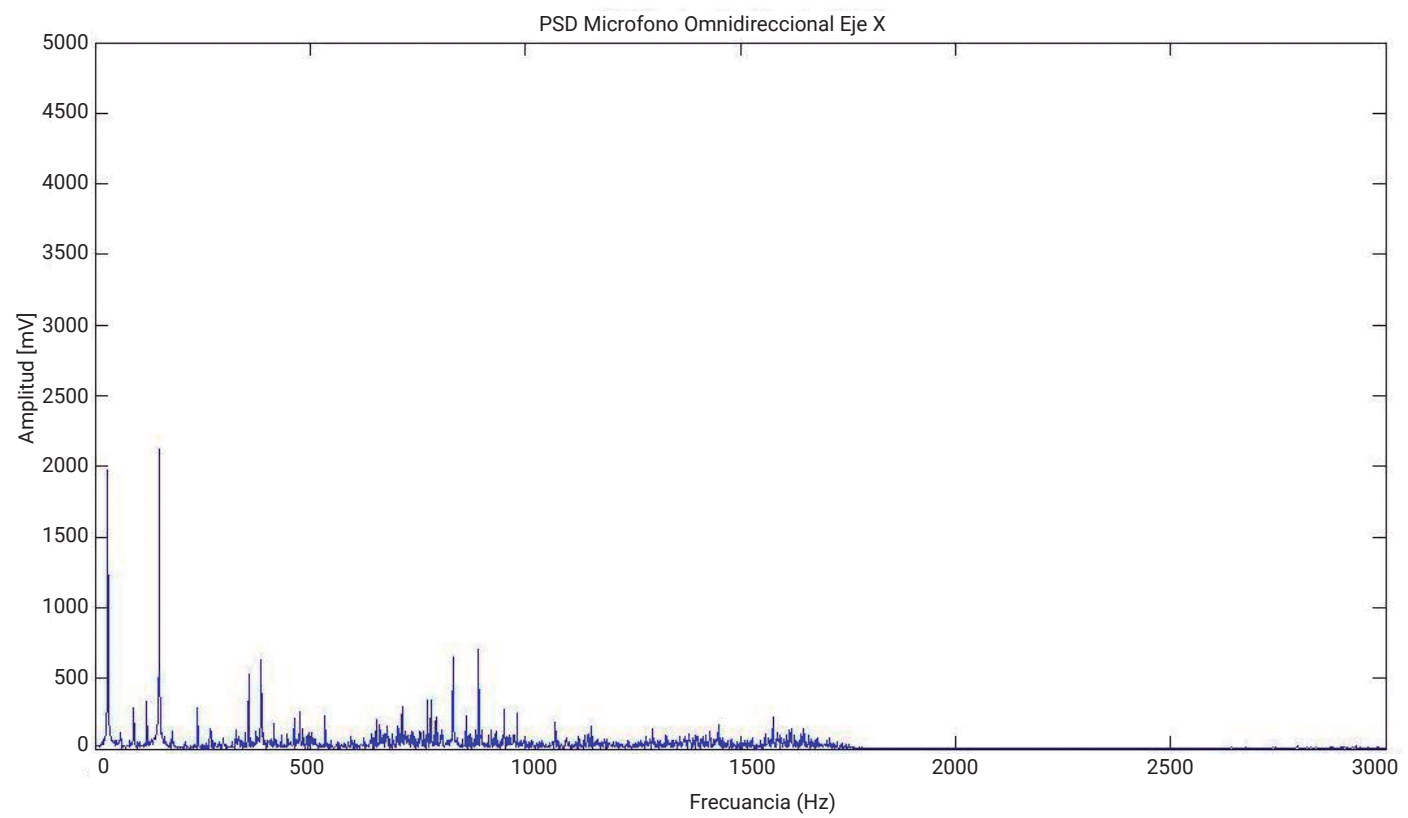

Fig. 5. Señal acústica horizontal del micrófono omnidireccional para la condición sin falla. Fuente: elaboración propia.

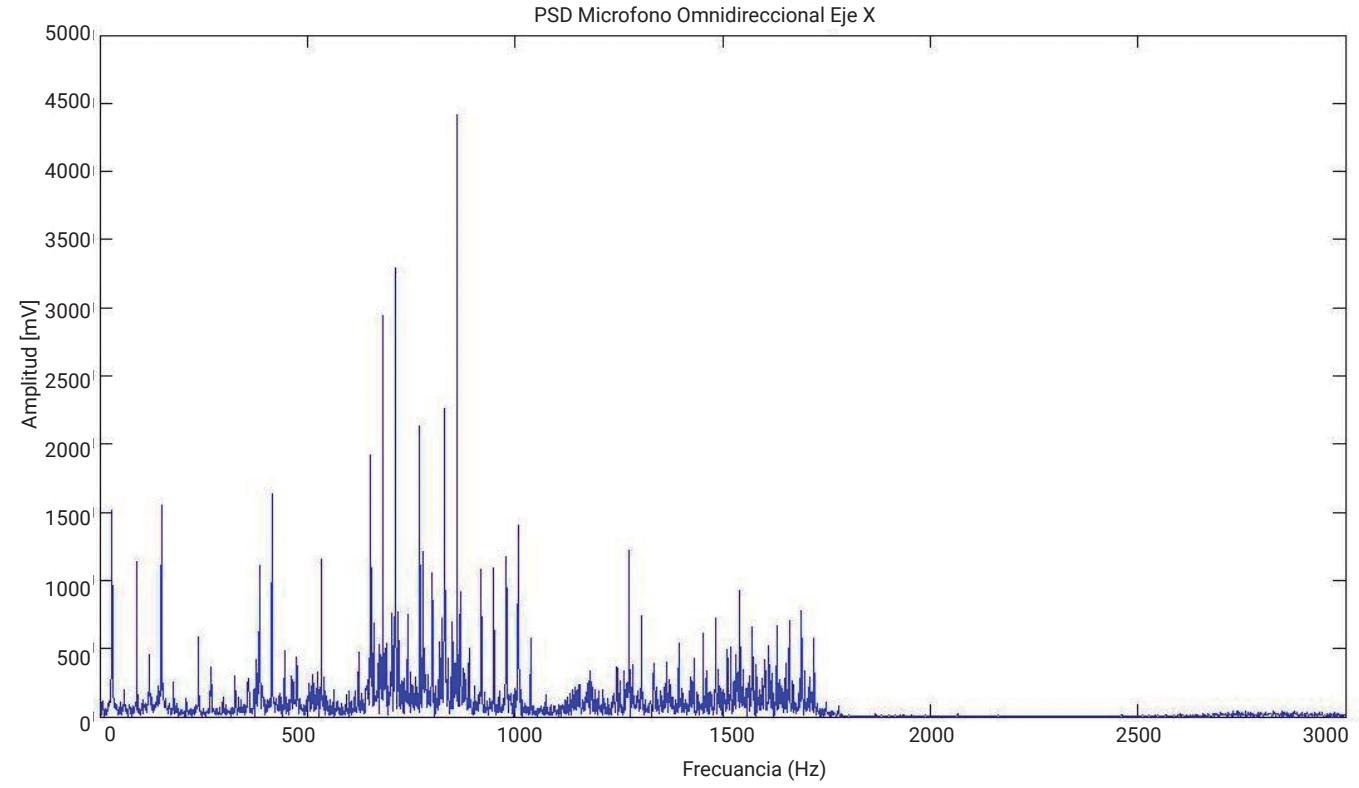

Fig. 6. Señal acústica horizontal del micrófono omnidireccional para la condición con falla Fuente: elaboración propia. 


\section{DISCUSIÓN Y ANÁLISIS DE RESULTADOS}

Se realizaron los análisis estadísticos de los ocho experimentos restantes y los resultados fueron los siguientes:

1. En el experimento 1 la variable de respuesta fue medida con un acelerómetro triaxial en el eje horizontal $(x)$, para la condición sin falla. A partir de esto se concluye que no hay factores significativos, lo que indica que no es importante el tipo de carga conectada y la variable de respuesta no es sensitiva al registro obtenido por el instrumento de medición. El nivel de confianza de la prueba estadística fue del $95 \%$.

2. En el experimento 2 la variable de respuesta fue medida con un acelerómetro triaxial en el eje vertical $(y)$, para la condición sin falla. Se concluye que es muy probable que la interacción no sea relevante, aspecto que muestra que no es importante el tipo de carga. Es significativa la interacción para un nivel de confianza del $95 \%$; sin embargo, no lo es para Esto demuestra que para establecer si realmente es significativa esta interacción, deberán realizarse más pruebas (réplicas) para la combinación de estas variables. No obstante, dado el comportamiento mostrado en las demás corridas de prueba y que para está prácticamente en el límite de significancia de la prueba $(4,41)$.

3. En el experimento 3 la variable de respuesta fue medida con un micrófono omnidireccional en el eje horizontal $(x)$, para la condición sin falla. Se concluye que no hay factores significativos, lo cual indica que no es importante el tipo de carga conectada y la variable de respuesta no es sensitiva al registro obtenido por el instrumento de medición. El nivel de confianza de la prueba estadística fue del $95 \%$.

4. En el experimento 4 la variable de respuesta fue medida con un micrófono omnidireccional en el eje vertical $(y)$, para la condición sin falla. Teniendo en cuenta lo anterior, se concluye que no hay factores significativos, lo que indica que no es importante el tipo de carga conectada y la variable de respuesta no es sensitiva al registro obtenido por el instrumento de medición. El nivel de confianza de la prueba estadística fue del $95 \%$.

5. En el experimento 5 la variable de respuesta fue medida con un acelerómetro triaxial en el eje horizontal $(x)$, para la condición con falla. De esta manera, se concluye que este tipo de transductor no es adecuado para la detección de la falla en las condiciones de prueba. Además, no se detectó que hubiese factores significativos, lo cual muestra que no es importante el tipo de carga conectada, y que la variable de respuesta no es sensitiva al registro obtenido por el instrumento de medición. En este caso el error tipo 1 (debido al cambio en la condición experimental) resultó mayor que el error aleatorio puro; lo que señala que la sensibilidad del instrumento de medición no es la adecuada bajo esta condición.

6. En el experimento 6 la variable de respuesta fue medida con un acelerómetro triaxial en el eje vertical $(x)$, para la condición con falla. Así, se concluye que este 
tipo de transductor no es adecuado para la detección de la falla en las condiciones de prueba. Además, no se detectó que hubiese factores significativos, lo que indica que no es importante el tipo de carga conectada y que la variable de respuesta no es sensitiva al registro obtenido por el instrumento de medición. En este caso el error tipo 1 (debido al cambio en la condición experimental) resultó mayor que el error aleatorio puro, lo que demuestra que la sensibilidad del instrumento de medición no es la adecuada bajo esta condición.

7. En el experimento 7 la variable de respuesta fue medida con un micrófono omnidireccional en el eje horizontal $(x)$, para la condición con falla. Se concluye que no hay factores significativos, lo que señala que no es importante el tipo de carga conectada y que la variable de respuesta no es sensitiva al registro obtenido por el instrumento de medición. En este caso la varianza del error aleatorio fue mayor que la del error primario (tipo 1), lo cual es siempre deseable. El nivel de confianza de la prueba estadística fue de $95 \%$.

8. En el experimento 8 la variable de respuesta fue medida con un micrófono omnidireccional en el eje vertical ( $y$ ), para la condición con falla. Se concluye entonces que es muy probable que la interacción no sea relevante, y esto demuestra que no es importante el tipo de carga. Es significativa la interacción para un nivel de confianza del $95 \%$; sin embargo, no lo es al $97,5 \%$ ni al $99 \%$ de confianza de la prueba, lo que indica que para establecer si realmente es significativa esta inte- racción, deberán realizarse más pruebas (réplicas) para establecer definitivamente el grado de significancia para la combinación de estas variables. Dadas las características del análisis de varianza y las condiciones físicas de la prueba, los resultados observados pueden deberse a que este tipo de transductores se hallan en el límite crítico de sensibilidad para estas condiciones, dado que la señal en la dirección vertical $(y)$ es mucho más intensa que en cualquiera de las otras dimensiones espaciales. Por tanto, antes de considerar adecuados estos micrófonos para detección de falla, habrá que hacer más pruebas confirmatorias.

\section{CONCLUSIONES}

Del análisis de los resultados puede deducirse que los acelerómetros no detectan diferencias estadísticas significativas para las condiciones sin falla y con falla para todas las combinaciones de cargas conectadas, razón por la cual este tipo de transductores no es una opción para la detección de una falla en la jaula del rodamiento a través del análisis de las señales de vibración, mientras que los micrófonos omnidireccionales muestran para las condiciones de prueba que son capaces de detectar una falla en la jaula del rodamiento de un generador síncrono, tanto en la dirección como en la dirección a partir de las señales acústicas emitidas por esta.

Se puede decir que, respecto al análisis de vibraciones de maquinaria rotativa, la frecuencia de operación está relacionada con la geometría del rodamiento que se emplee, así como con la velocidad de operación de 
estas, por lo que conforme se modifique la velocidad van cambiando las frecuencias características de falla en los rodamientos (por ejemplo, a $3600 \mathrm{rpm}$ se tendrían valores de $B P F I=267,297 \mathrm{~Hz}, B P F O=152,703 \mathrm{~Hz}$, $F T F=21,815, B S F=101,768$ y defectos en las bolas $=203,535 \mathrm{~Hz}$ ).

Finalmente, se concluye que el análisis acústico puede utilizarse de manera confiable en cualquier sistema mecánico que utilice balero o rodamiento en su funcionamiento.

\section{REFERENCIAS}

[1] Ereneovable.com, "La energía eólica en el mundo," 2015. [En línea]. Disponible en: https://erenovable.com/aerogeneradores-eolicos/energia-\%20 eolica/

[2] A. Suárez. Idear. Análisis de fallas en rodamientos. Buenos Aires, Argentina: Soluciones Idear, 1998.

[3] G. White. Introducción al análisis de vibraciones. Woburn, MA, Estados Unidos: Ázima DLI, 1990-2010.

[4] J. A. Royo, G. Rabanaque y F. Torres, "Análisis de vibraciones e interpretación de datos," 2012, [En línea]. Disponible en: http://guemisa.com/articul/ pdf/vibraciones.pdf. [URL desabilitado]

[5] M. L. Sin, W. L. Soong y L. N. Ertugrul, "Induction machine on-line condition monitoring and fault diagnosis - A survey," en Australasian Universities Power Engineering Conf., Christchurch, New Zealand, 2003.
[6] Z. Medrano, C. Pérez, M. A. Armas y C. Amaro, "Un estudio sobre la localización, detección y diagnóstico de fallas en máquinas eléctricas," Ciencia e Ingeniería Neogranadina, vol. 23, no. 1, pp. 37-59, 2013, https://doi.org/10.18359/ rcin. 231

[7] Z. Medrano, T. Pérez y S. Gómez, "A review on detection and fault diagnosis in induction machines," Publicaciones en Ciencias y Tecnología, vol. 8, no.1, pp. 11-30, 2014. [En línea]. Disponible en: http://bibvirtual.ucla.edu.ve/ $\mathrm{db} / \mathrm{psm}$ _ucla/edocs/pcyt/pcyt08/ pcyt080101.pdf. [URL desabilitado]

[8] Agco Corporation y P. Matthers, "Acoustic fault detection of mechanical systems with active noise cancellation," Patent application no. 20130182865, 2013, [En línea], Disponible en: http:// www.patent sencyclopedia.com/ app/20130182865\# ixzz 3SDtPXKxL

[9] P. J. Ross. Taguchi Technique for Quality Engineering. Nueva York, Estados Unidos: McGraw-Hill, 1995,

[10] Z. Y. Medrano, C. Pérez, J. Gómez y M. Vera, "Nueva metodología de diagnóstico de fallas en rodamientos en una máquina síncrona mediante el procesamiento de señales vibro-acústicas empleando análisis de densidad de potencia," Ingeniería Investigación y Tecnología, vol. 17, no.1, pp 7385, 2016. https://doi.org/10.1016/j. riit.2016.01.007

[11] H. Usgame, C. Pedraza y J. Quiroga, "Acoustic emission-based early fault detection in tapered roller bearings." 
Ingeniería e Investigación, vol. 33, no.3, pp.5-10, 2013,

[12] ALL-TEST Pro, LLC, "Detectando fallas en rodamientos utilizando métodos de prueba eléctricos y mecánicos de vibración," 2009. [En línea]. Disponible en: http://confiabilidad.net/assets/ uploads/art/PDF/detectando_fallas_ en_rodamientos_atp.pdf

[13] FAG Sales Europe-Iberia, "Averías de los rodamientos. Reconocimiento de daños e inspección de rodamientos," Barcelona, 2003, [En línea]. Disponible en: http://217.160.235.229/ PDF-Arizti/wl_82102_2_es_es.pdf

[14] Energiza, "Análisis de vibraciones: Una tecnología clave del mantenimiento predictivo", 2011. [En línea]. Disponible en: http://www.energiza.org/mantenimiento-de-plantas/19-mantenimiento-de-plantas/516-analisis-de-vibra- ciones-una-tecnologia-clave-del-mantenimiento-predictivo

[15] SKF, "Daños en los rodamientos," 2016. [En línea]. Disponible en: http:// www.skf.com/co/products/bearings-units-housings/roller-bearings/ principles/troubleshooting/bearing-failures-and-their-causes/bearing-damage/index.html

[16] V. Wowk. Machinery Vibration-Measurement and Analysis. Nueva York, Estados Unidos: McGraw-Hill, 1991,

[17] SKF, "Frequency calculator-Bearing frequenciescalculation,"2016. [Enlínea]. Disponible en: http://www.skf.com/ group/knowledge-centre/engineeringtools/skffrequencycalculator.html

[18] H. G. Pulido y R. de la Vara. Análisis y diseño de experimentos. México, D.F., México: McGraw-Hill, 2008. 